Fecha de recepción: marzo 2020 Fecha de aceptación: abril 2020 Versión final: mayo 2020

\section{Economía distribuida en la Enseñanza del Diseño}

Marco Vinicio Ferruzca Navarro ${ }^{(1)}$ y

Sergio Dávila Urrutia ${ }^{(2)}$

Resumen: Diseñar para las personas, para su entorno y su situación significa cambiar también el enfoque de las cosas a los humanos, sus experiencias y su contexto. Es necesario pasar de un cambio de desarrollo inconsciente, es decir sólo por aumento de la productividad, a consciente considerando los ciclos de los elementos y su impacto a futuro. Las carreras de diseño de igual manera han sufrido un cambio de enfoque del objeto al sujeto. La complejidad de los problemas da más posibilidades de desarrollo al diseñador, pero los límites de la disciplina son más difusos. En este trabajo se explora el vínculo de la enseñanza del diseño, con la economía distribuida a partir de los conceptos propuestos por Ezio Manzini, la teoría emergente de Brown y el pensamiento exponencial de Diamandis. Para ello, se revisan varias experiencias de ejercicios de diseño. La intención es lograr un mejor entendimiento de cómo estos conceptos contribuyen a proyectar soluciones de diseño más estratégicas ad hoc a nuevos modelos de desarrollo como el de economías distribuidas.

Palabras clave: Carreras de diseño - objeto de diseño - sujeto de diseño - economía distribuida

[Resúmenes en inglés y portugués en las páginas 177-178]

(1) Ph.D., 2008 The Barcelona Tech (Polytechnic University of Catalonia), School of Industrial Engineers (Barcelone, Spain). Posgraduate, 2011 en Telefónica Learning Services \& The Barcelona Tech, Field of Study Business, Design and Technology Field of Study Multimedia Engineering \& Virtual Reality. Actualmente, es Director de la División de Ciencias y Artes para el Diseñode la Universidad Autónoma Metropolitana, México

(2) Master of Arts by the Aalto University in Helsinki, Finland. Diseñador Industrial interesado en nuevas tecnologías y fabricación post-industrial. Profesor-investigador de la Universidad Autónoma Metropolitana, México. Experiencia en prototipado rápido e impresión 3D. 


\section{Introducción}

A lo largo de la historia de las carreras de diseño en nuestra universidad se ha visto un cambio de enfoque del objeto al sujeto. La formación de diseñadores se centraba anteriormente en un reto de diseño concreto que en su planteamiento contenía la respuesta. Al pedirle a un alumno diseñar un juguete, una silla, una luminaria, un punto de venta, la respuesta estaba contenida en el reto y las posibilidades de diseño eran muy acotadas. La implicación más fuerte de esta situación es que se reduce la capacidad de investigar de los alumnos. Eventualmente se empezó a ampliar el panorama a problemas más complejos y enfocados en una comunidad, por ejemplo: de diseñar un juguete - a diseñar un juguete para niños con discapacidad - a diseñar un sistema lúdico de estimulación a niños con discapacidad. El enfoque al ampliarse tiene mayores áreas en donde el diseño puede influenciar. Centrarse en las personas hace que no sólo el diseñador considere los objetos con los que se relacionan las personas y sus funciones de uso, sino también el cómo accede a estos objetos, cómo los adquiere, cómo los mantiene, qué sistemas de apoyo existen para que realice la tarea que busca realizar, cómo la experiencia alrededor de la oferta se vuelve cautivadora, significativa, transformadora, y cómo eventualmente deshecha o prescinde de los productos y servicios. Esta apertura da posibilidad al diseñador de tener injerencia en toda la rutina del usuario, en el tiempo de vida de los productos y servicios, y en el manejo cíclico de los recursos.

El aumento en la complejidad de los retos le brinda más responsabilidades y posibilidades de desarrollo profesional al diseñador, no obstante, también hace más difusos los límites de la disciplina, los límites entre producto y servicio, los límites entre físico y virtual, los límites entre lo tangible y lo tangible, los límites entre experiencia y pertenencia, etc. El diseñador sigue siendo este eje profesional que busca darle sentido a los espacios y los objetos, redefinir el significado ante este escenario de cambio y límites difusos. El diseñador necesita ser resiliente, adaptarse rápidamente al cambio y buscar nuevas definiciones que puedan hacer sentido para las personas al confrontar los retos de nuestros tiempos.

En el terreno de la enseñanza en diseño y en específico de la experiencia docente que se presenta a través de los ejercicios de diseño que se reportan más adelante, el diseño para la innovación social es un tema que se trabaja desde diferentes ángulos a lo largo del proceso de formación del estudiante de diseño. Lo entendemos como las nuevas ideas (productos, servicios y modelos) que simultáneamente atienden necesidades sociales y generan nuevas relaciones, valores y colaboraciones. Más allá de ser una propuesta que desde el diseño intenta resolver una problemática compleja, se convierte en un entendimiento profundo de un grupo de personas para desde una perspectiva humana buscar resolver las necesidades, anhelos y deseos apoyados con métodos y herramientas del diseño. En este tipo de propuestas el diseñador no buscará ser el líder del proyecto sino la partera de las soluciones, una partera no hace todo el trabajo, pero está ahí todo el tiempo para apoyar a la mejor gestión (Brown, 2018). En un esquema de diseño para la innovación social se busca distribuir las responsabilidades creativas entre los participantes, así como las responsabilidades de aplicación de soluciones y hasta la creación de una economía distribuida que en lugar de depender de un sistema central busca generar nuevos sistemas económicos o sistemas distribuidos. 
El diseño es una cultura y una práctica que concierne el orden de las cosas para obtener el significado y las funciones deseadas. Involucra a los agentes a participar y co-crear. El rol de los diseñadores es detonar y apoyar estos procesos abiertos de colaboración, usando sus habilidades para concebir y empoderar las iniciativas ante las problemáticas.

El presente trabajo se organiza de la siguiente manera: en primer lugar se hace una breve revisión del panorama actual del diseño; en segundo lugar se revisan los conceptos de economía distribuida, teoría de las estrategias emergentes y pensamiento exponencial; en tercer lugar, se describen los resultados de un par de ejercicios académicos de diseño que sirven como base para un análisis de la enseñanza del diseño a partir de los conceptos teóricos revisados y se esbozan algunas conclusiones sobre su repercusión.

\section{Marco teórico}

\subsection{El escenario actual del diseño}

El diseño ha sido considerado en relación con el desarrollo de nuevos productos, pero las definiciones más actuales tratan de extenderlo a nuevos elementos con los que tiene potencial de lidiar hoy en día. Según Manzini (2015), el interés en el campo del diseño ha cambiado de productos a relaciones (como interacciones sociales, servicios, comunicación), Son organizaciones socio técnicas las que el diseño está contribuyendo a construir. Existe un amplio consenso entre los expertos sobre el potencial que tiene la innovación social para modificar muchos de los patrones que nos perjudican como sociedad. Sin embargo, como señala Manzini, una nueva cultura y práctica son requeridas. Es aquí en donde el diseño tiene una oportunidad de potenciar la innovación social, siempre y cuando su raíz ontológica cambie para convertirse en una actividad más abstracta, y por tanto amplía, de tal manera que pueda interactuar con mayor claridad con los sistemas socio técnicos en los que estamos inmersos.

El diseño es una profesión al servicio de las necesidades humanas. Reconoce que los problemas se pueden convertir en oportunidades, por lo que busca contextualizarlos y seguir procesos complejos que idealmente produzcan un sistema de productos y servicios, una estrategia de organización y estructuras para producir organizaciones. A diferencia de las ciencias sociales, que nos ayudan a entender el comportamiento de las personas, sus motivaciones y dar fundamento cultural e histórico a la naturaleza del actuar de sus comunidades. Sin embargo, su campo de acción se queda en el análisis. El diseño por su naturaleza es práctico, y busca entender a las personas y su situación con el fin de proponer una serie de elementos tangibles que puedan apoyarlos a mejorar su situación. Bajo esta visión se podría definir al Diseño como ciencia del comportamiento aplicado, como un agente en el cambio de comportamiento en las personas.

Ante este escenario, y el evidente surgimiento de una nueva epistemología del diseño, destacan los siguientes elementos:

- Existen límites cada vez más borrosos entre productos y servicios, entre físico y digital, entre pertenencia y experiencia. 
- Se están deconstruyendo muchos límites entre empresa, organización, comunidad, familia, relaciones.

- Existe un mayor enfoque hacia las expectativas, demandas, anhelos y deseos de las personas. - Las tecnologías actuales tienden a la producción distribuida. Es más fácil encontrar un servicio de corte $\mathrm{CNC}$ o de impresión $3 \mathrm{~d}$ cerca de casa que un servicio de rotomoldeo o termoformado.

Todo esto implica un cambio hacia la definición de lo "Industrial". Tal vez más que un cambio, es un regreso a su etimología: Indos=dentro, Struo= construir. Es decir, construir desde dentro, imaginar, concebir, proyectar. Esto libera también el pensamiento creativo del diseñador a no centrarse en la sobreproducción, en el crecimiento económico sino en la construcción del tejido social, la intrincada relación de personas, objetos, espacios, y emociones. La civilización es un tejido de valores y creencias que permea entre sus miembros. Si se sigue definiendo al diseño industrial como diseño para la industria pierde la posibilidad de aplicación en políticas gubernamentales, redefinición del espacio público, generación de organizaciones civiles, diseño fuera del sistema económico o hasta diseñar nuevos sistemas (no necesariamente económicos).

Las habilidades del diseñador incluyen dar sentido a las cosas y hacer un sistema de significado a su alrededor (Manzini, 2015). Experimentar con las relaciones humanas. Buscar nuevas formas de colaborar, vivir y producir. Tomar la sociedad como laboratorio. No por dejar de lado la responsabilidad hacia los seres humanos, sino porque se está consciente de que el trabajo nunca está terminado. Las iniciativas sociales se entienden como constantes prototipos en mejora. Investigación mediante la acción. No es ingeniería social para hacer eficiente la productividad del individuo, es diseño para la innovación social la cual tiene el fin de generar bienestar para las personas.

El cambio de enfoque para abandonar la productividad como objetivo principal nos abre la posibilidad a experimentar con distintos modelos alternativos. Si el objetivo es generar bienestar en la comunidad que se está atendiendo tal vez se debería de reactivar la economía local de esa comunidad de forma sustentable, es decir que pueda perdurar y afectar en lo mínimo a las relaciones humanas, al consumo de materias primas y el impacto ambiental que podría tener, por ejemplo, el transporte de bienes de una comunidad a otra. En otras palabras, los tres ejes del diseño sustentable: financieramente viable, ambientalmente factible, y socialmente deseable.

Es entonces que la visión de Manzini conecta diferentes teorías en su mapa de modos de diseño. El cual explora a fondo en el libro "Diseñar, cuando todo el mundo diseña", que con tan atinado título busca compartir que el diseño no es una herramienta de los dioses licenciados del Olimpo, sino que es una caja de herramientas que cualquier interesado puede probar, si no es que ya lo ha hecho anteriormente. Esto más allá de quitarle responsabilidades al profesionista de diseño o robarle su materia de trabajo, se muestra como una posibilidad de empoderamiento a las comunidades que tienen acceso limitado a bienes y servicios. La distribución de responsabilidades no es una manera de desmantelar la profesión del diseño, por el contrario, es la manera en que naturalmente se han organizado los grupos humanos antes de el credo de la globalización y las supercherías de la economía. 
Es natural que los miembros de una comunidad tomen responsabilidad de mejorar su condición. Rigoberta Menchú solía decir: Sólo los privilegiados se detienen en la crítica, los demás tenemos que buscar soluciones. Refiriéndose a que es cómodo no involucrarse. $\mathrm{Al}$ hablar de responsabilidades distribuidas implica, por ende, el involucramiento de los miembros de la comunidad.

¿Cómo se diseñan situaciones sociales en donde la gente se involucre y empodere para generar soluciones? Al respecto, algunos expertos proponen promover un diseño para la innovación social a través del cual se generan nuevas ideas (productos, servicios y modelos) que simultáneamente atienden necesidades sociales y generan nuevas relaciones y/o colaboraciones. Son útiles para la sociedad e incrementan la capacidad de resolver situaciones en los miembros de una sociedad.

A los gobiernos se les acaban las ideas, la forma clásica de hacer políticas públicas no resuelve la complejidad de los grandes problemas de nuestros tiempos. El diseño para la innovación social considera a las personas en el problema como posibles agentes de sus soluciones y al diseñador como orquestador de las soluciones.

Adicionalmente, otra idea que ha surgido vinculante a promover un diseño para la innovación social es el concepto de sistema distribuido y resiliente. La lógica es que este tipo de sistemas son menos vulnerables que los sistemas centralizados. En el sistema centralizado todo se cae al fallar el nodo central ya que el sistema depende de él. Un sistema descentralizado busca atacar esta problemática generando diferentes puntos de convergencia que puedan resistir o mantenerse aun cuando uno de los nodos centrales falle. Sin embargo, la forma más resiliente en que puede presentarse un sistema es cuando se distribuyen las redes. La complejidad de interconexión es mayor, pero al momento de fallar alguno de sus puntos de convergencia el sistema no sólo sigue funcionando, sino que se puede reparar más fácilmente.

Bajo esta perspectiva de sistemas distribuidos es que se intenta replicar también este concepto en las diferentes esferas sociales. A saber infraestructura distribuida, redes alimenticias distribuidas, fabricación y economía distribuidas, siendo esta última uno de los aspectos a desarrollar en la siguiente sección.

\subsection{Economías Distribuidas}

El concepto de economía distribuida (Johansson et al., 2004) en su propuesta original debe ser entendida como una estrategia para guiar el desarrollo industrial de manera sustentable. Para ello es necesaria una transformación en las dinámicas sociales, económicas y ambientales orientadas por la economía neoliberal.

En una economía distribuida, un porcentaje de los procesos de producción son distribuidos entre diferentes unidades de producción dentro de una región. Se trata por lo tanto de empezar de manera discreta (pequeña) a nivel regional y sacar provecho del entusiasmo individual, así como del espíritu emprendedor con una vista de crecimiento. No se basa en un número de unidades de producción, sino de una red interregional que promueve la producción de baja escala, con una cadena de suministro y recursos locales de acuerdo con las necesidades locales (Lizarralde, 2018). 
Los principios de este tipo de economías se basan en:

- Creación de riqueza para un amplio número de personas.

- Reinventar la calidad y priorizar la antes de la eficiencia en producción.

- Horizontalidad e innovación abierta.

- Sistemas flexibles de producción de baja escala.

- Diversificación de necesidades y deseos.

- Relaciones simbióticas.

- Diversidad ecológica, económica y social.

- Calidad de vida como un componente integrado para el desarrollo y la innovación.

- Nuevas relaciones entre el productor-consumidor.

- Innovación y diseño integrados

- Capital ecológico y social como una ventaja

- Una nueva relación entre sistemas de producción de gran y pequeña escalas.

- Espíritu colectivo y colaboración

- Intercambio de recursos intra e interregional.

Desde la óptica de Manzini, la moneda es solo un símbolo de valor, el papel moneda no tiene un valor real para el ser humano más que lo que representa. Y éste representa el poder adquisitivo, o como diría Pasolini "El fantasma del Mercantilismo". La idea de que, si uno necesita comer, puede pagar por alimento, si uno necesita techo puede pagar una renta, si uno necesita transportarse puede adquirir un automóvil, y así interminablemente. Las economías distribuidas tratan de generar su propio símbolo de valor localmente, tal vez a través de su forma más obvia: el trueque de productos y servicios. Las economías de intercambio tratan de mantener el valor comercial dentro de la comunidad, de la misma manera que lo haría un centro de videojuegos al vender su propia moneda para cada máquina y así poderse quedar con los remanentes. Y de la misma manera que la economía permea en todos los aspectos de la cultura y sociedad occidental, los beneficios de las economías distribuidas tienen el potencial de afectar todos los aspectos de nuestra vida contemporánea, mejorando y fortaleciendo las relaciones humanas, aprovechando los talentos propios de cada individuo, y generando bienestar común al darle propósito y valor al trabajo de cada persona. De la misma manera que un niño intercambia sus dulces de Halloween o su juguete repetido, las redes sociales han abierto la posibilidad de intercambiar productos y oficios. Las redes más comerciales buscarán siempre que haya un intercambio monetario para poder quedarse con un porcentaje así que el verdadero valor en este caso lo tienen las redes autogestionadas de personas dentro de una comunidad. El ejemplo de Manzini, es un caso cerca del Politécnico de Milán en donde los adultos mayores intercambian una recámara de su casa a los estudiantes por tiempo para ayudarles con las labores domésticas. Generando no sólo redes de apoyo entre la fuerza vital de la juventud y los ancianos, sino intercambio de saberes en una sociedad como la europea que tiende a ser muy horizontal generacionalmente en sus redes. 


\subsection{Teoría de estrategias emergentes}

Como una respuesta por intentar entender mejor los problemas actuales que enfrenta la sociedad y dar forma a un mejor futuro, Brown (2017) propone un marco teórico, denominado Estrategia Emergente, útil para identificar y valorar las estructuras materiales y no materiales que hacen nuestras mentes y cuerpos estén en constante cambio. Parafraseando a Nick Oblensky en (Brown, 2017), lo emergente es la forma en que los sistemas y patrones complejos surgen de una multiplicidad de interacciones relativamente simples.

En este escenario, el todo es un espejo de las partes, lo emergente está más allá de la suma de sus partes. En un estado de emergencia, entendido como sistema, se hace uso de todo en un proceso iterativo. Por lo tanto, una estrategia emergente puede ser una vía para practicar la complejidad.

De manera más simple, para Brown, una estrategia emergente puede ser entendida como las maneras en que los seres humanos practican estar en armonía con otros y con nuestro hogar, practican la complejidad, y crece un futuro convincente a través de interacciones simples. Se trata por lo tanto de cambios intencionados que aumentan nuestra capacidad de encarnar los mundos justos y liberados que anhelamos.

Este marco teórico propone siete elementos que pueden ser aplicados para entender sistemas complejos:

- Fractal. En referencia a la relación entre lo pequeño y lo grande. Todo guarda una relación, hay patrones en todo el sistema del que formamos parte y a todos los niveles.

- Adaptativo. Orientado a los procesos de cambio para ajustarse a un proceso o situación. Este elemento es fundamental porque representa el comportamiento humano frente al cambio constante y por lo tanto desvela las intenciones estratégicas de cambio.

- Interdependencia y descentralización. Responde a las preguntas quiénes somos y cómo compartimos, en pro de una sociedad más interdependiente, de liderazgo compartido y confianza mutua.

- No lineal e iterativo. El ritmo y los patrones de cambio. La transformación no es un proceso lineal.

- Resiliencia y justifica transformadora. Cómo nos recuperamos y nos transformamos.

- Crear más posibilidades. Cómo nos movemos hacia la vida.

\subsection{Crecimiento exponencial}

A partir de la premisa que indica la sociedad está enfrentando grandes retos y por lo tanto también se requieren grandes cambios en la sociedad, es necesario señalar que se requieren de soluciones exponenciales que nacen de un pensamiento exponencial (Hoffman, 2018). Al respecto, la evolución de la tecnología digital es la muestra de un cambio exponencial y no linear que de igual manera demanda este nuevo tipo de pensamiento que es más disruptivo y ocasiona que mucho de los modelos de negocio vigentes tiendan a caducar de manera más rápida (Sörman-Nilsson, 2013). La idea de un pensamiento ex- 
ponencial puede ser entendida a partir de la Ley de Moore que tanto se ha utilizado para explicar la evolución tecnológica.

Diamandis y Kotler (2016) han desarrollado una estructura conceptual que ayuda entender mejor la evolución exponencial de la tecnología, denominada "las seis D": digitalización, decepción, disrupción, desmonetización, desmaterialización y democratización. Ver Tabla 1.

\begin{tabular}{|c|c|c|}
\hline Las seis " $D$ " & Descripción & Ejemplo \\
\hline Digitalización & $\begin{array}{l}\text { Transición de un producto o proceso de } \\
\text { físico a digital, con pos ib ilidades de } \\
\text { evolucionar de mane a exponencial. }\end{array}$ & $\begin{array}{l}\text { Las fotografís y su proceso de revelado pesaron } \\
\text { de un formato fisioo a uno digital. }\end{array}$ \\
\hline Decepción & $\begin{array}{l}\text { Periocb dura nte el cual el crecimiento } \\
\text { exponencial pasa casi desapercibido. }\end{array}$ & $\begin{array}{l}\text { Las capacickack de almacena mie nto fueron } \\
\text { creciendo progresivamentesinser notacks lasta } \\
\text { que aka nzalon gra ndes niveles. }\end{array}$ \\
\hline Disrupción & $\begin{array}{l}\text { Gene ración de nuevos mercados y } \\
\text { desaparición de otros como consecuencia } \\
\text { de una tecno bgía dis ruptiva. }\end{array}$ & $\begin{array}{l}\text { Servicios como Netfliø@ Spotify@ han } \\
\text { desphazado otros negocios tradicio males a pa rit } \\
\text { de sus innovaciones tecnobigicas y de servicio. }\end{array}$ \\
\hline Desmonetización & $\begin{array}{l}\text { Remoción del dinero que se paga por el } \\
\text { uso de bienes y servicios. }\end{array}$ & Modebs de negocio FREEMIUI, tipo Google. \\
\hline Desmaterialización & Remoción de los bienes y los servicios. & $\begin{array}{l}\text { Desa parición de la cá mala y sustitución porel } \\
\text { teléfono inteligente. }\end{array}$ \\
\hline Democratización & $\begin{array}{l}\text { Final de una reaoción en cadera } \\
\text { exponencial, el resultado de las últimas } \\
\text { dos D. }\end{array}$ & $\begin{array}{l}\text { Los planes de Face booke por distribuir el acoeso } \\
\text { a internet a cada ser huma no que rabita k tierra. }\end{array}$ \\
\hline
\end{tabular}

Tabla 1. Las seis “D” propuestas por Diamandis y Kotler

De fondo, la utilidad de esta estructura conceptual radica en la posibilidad de entender por qué muchas compañías o industrias completas están desapareciendo. Véase el caso del transporte público, los consumidores de este tipo de servicios se inclinan por los nuevos procesos, digitales principalmente, para hacer uso de ellos. Uber $\odot$, Cabify@ u otro tipo de plataformas que ofrecen soluciones de transporte han desplazado a los taxistas en diferentes regiones del mundo. Con este tipo de soluciones, los procesos tradicionales para poder tomar un auto se fueron digitalizando, mientras la tecnología que los soporta maduraba. Hoy estas plataformas digitales comparten una buena parte del mercado de transporte público, afectando al gremio de los taxistas tradicionales. Adicionalmente, la remoción del dinero en este tipo de plataformas es evidente si se considera que el pago de este tipo de servicios se da con el apoyo de la banca digital. Desaparecen muchos vehículos de uso público como los taxis, se desmaterializan, pero irónicamente aparecen otros de uso particular que contribuyen a la democratización de este servicio soportado por tecnología exponencial. 
En otro ámbito, la manufactura también está sujeta a muchas disrupciones. Véase el caso de la industria automotriz, la aeroespacial, el sector de equipos médicos, entre otras, en donde la tecnología de impresión 3D ha venido a cambiar sus modelos de negocio. De igual manera, otras tecnologías como los sensores y redes, la inteligencia artificial y la biología sintética, están provocando una evolución exponencial en muchos de las actividades que desarrollamos. Cada una de estas tecnologías exponenciales puede ser entendida a través del marco teórico de las 6D.

\subsection{Estructura epistemológica y casos de estudio}

Con el fin de realizar una primera aproximación para valorar en qué medida los conceptos de economías distribuidas, estrategia emergente y pensamiento exponencial están presentes en el proceso de diseño, y en particular en la educación en diseño, ha sido necesario elaborar una primera representación que sirva de guía para tal propósito. Véase la figura 1.

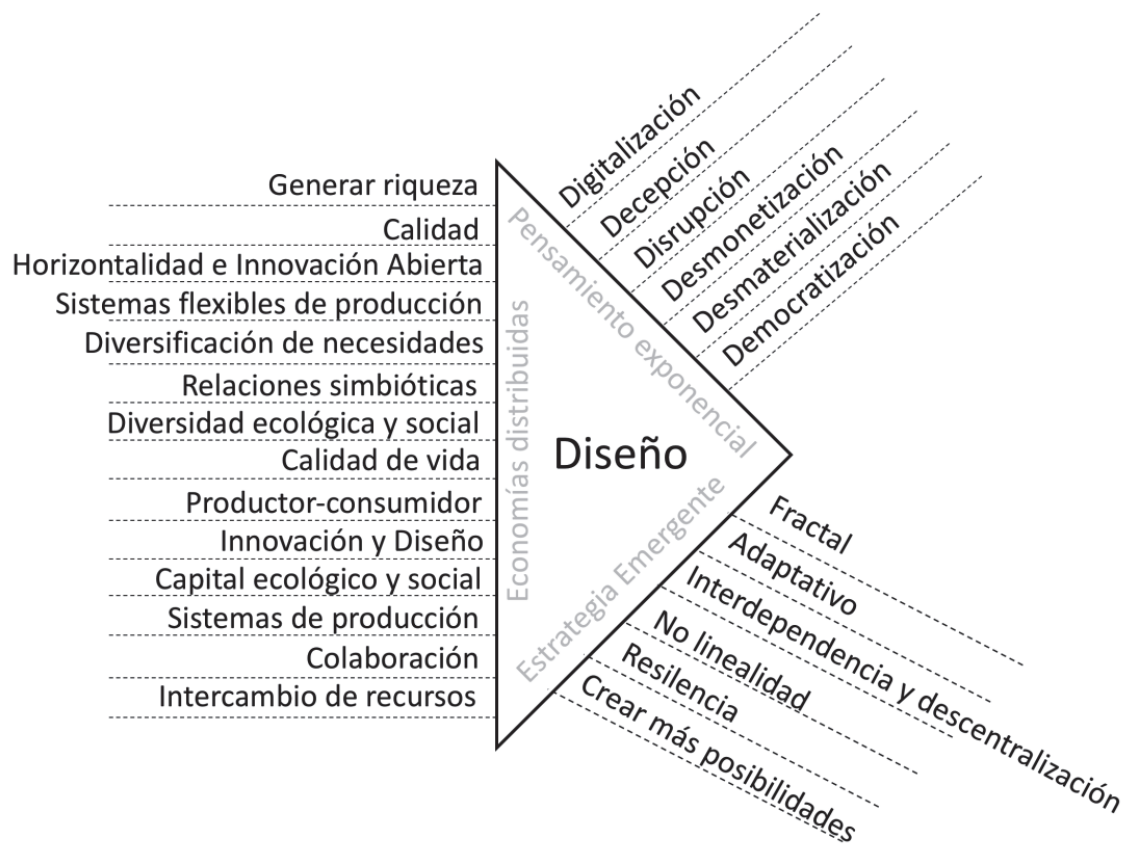

Figura 1. Elementos clave de los conceptos economías distribuidas, pensamiento exponencial y estrategia emergente. 


\section{Análisis situado de la Enseñanza en Diseño: el caso de un curso práctico de diseño industrial.}

En esta sección se presentan dos ejercicios escolares elaborados por estudiantes de la carrera en diseño industrial de una universidad pública en el marco de un curso con orientación proyectual. La intención es identificar la presencia de los conceptos teóricos anteriormente descritos y desarrollar un mejor entendimiento sobre la importancia de permear en la enseñanza del diseño la idea de economías distribuidas. Por lo tanto, en esta ocasión el principio que adoptan los autores de este artículo es de carácter exploratorio, convencidos dela importancia que tiene para el diseño contribuir con el desarrollo de sistemas producto-servicio sustentables, pero sobre todo de la formación de diseñadores comprometidos en la búsqueda de soluciones a los problemas reales de la sociedad.

Hay que aclarar que la idea de repartir responsabilidades entre los integrantes de una comunidad que da forma a una economía distribuida no es nueva, parte también de replicar ejemplos en el arte participativo, lo que se denominó en su momento como "Diseño Abierto" (Atkinson, 2011), en donde el usuario en participar, completa la obra de arte. Cuando el Artista Liam Gillick decía que su arte es como la luz del refrigerador, sólo se activa cuando la gente lo abre, o de lo contrario su arte son sólo cosas en un cuarto. Más allá de la triada semiológica, en este concepto el participante, la audiencia y el denominado "espectActor" (Boal, 1998) completan la estética de la obra. En esa misma tonalidad Paul Atkinson (2011) habla de que el diseño se completa cuando el usuario, experto en su problemática, completa el proceso de diseño. Desde esa aproximación de participación en la obra ya existe un sentido de involucrar a las personas en el proceso y de que todo mundo puede diseñar. Ahora en el sentido de la economía distribuida se busca involucrar a la comunidad a tejer la red de recursos y activarla.

Estos conceptos se exploran y profundizan en el curso durante el cual se realizaron los ejercicios aquí reportados, a partir de facilitar una visión al alumno del potencial que tiene el diseño para detonar y establecer mejoras en el tejido social. La duración de este curso es de12 semanas, de las cuales 8 se dedican a estudiar a la comunidad, a utilizar diversas herramientas como el safari de usuarios, la entrevista, el "shadowing", y como cúspide de esta investigación de usuario se diseñan sondas culturales y se lanzan al contexto del usuario para recopilar evidencia a distancia (Kumar, 2013). Estas herramientas otorgan un universo de información al diseñador el cual identifica oportunidades individuales, sistémicas y estratégicas que devienen en un sistema de productos y servicios que pueden aportar mejoras, soluciones y alternativas al contexto.

Los problemas de una comunidad son usualmente complejos y no pueden solucionarse de tajo con un nuevo objeto que aparezca en su entorno, por eso este enfoque estratégico se propone como un libro de jugadas en donde pueden existir objetos, pero no aparecen sólo de tajo, algunos toman tiempo en gestarse, otros se construyen por la comunidad, algunos otros van respaldados por un servicio, otros necesitan rodearse de un espacio que los apoye, iluminación, señalética, etc. Estas diferentes jugadas se reúnen en una compilación de estas a la que llamamos "Playbook" y contiene un análisis de contexto y posibles estrategias a seguir para mejorarlo. 
Los sistemas de producto servicio son una manera de dar solución a una serie de problemáticas complejas dentro de un contexto social. Si el problema fuera simple tal vez la solución podría ser igual de simple, pero al presentarse una serie de problemáticas interrelacionadas en un ecosistema de personas, artefactos, emociones e identidades, las soluciones no pueden ser simples. Se necesitan diferentes jugadas para cada momento, de la misma manera que el entrenador del equipo de basquetbol tiene un libro de jugadas las cuales comparte con el equipo y los invita a realizarlas de acuerdo con cómo vaya leyendo el partido, de esa misma manera se busca leer el contexto social y desarrollar un libro de estrategias emergentes para las situaciones que se puedan dar.

En uno de los casos que se exponen a continuación los estudiantes se dieron cuenta al analizar el contexto que la solución para un parque no era poner más juegos infantiles pues eran usados comúnmente por jóvenes con problemas de drogas. La solución consistía en diferentes estrategias, desde demarcar zonas distintas, proponer señalética y carteles con información sobre el efecto dañino de las drogas, solicitar cuerpos de seguridad en horarios clave, aumentar la iluminación, hacer espacios de esparcimiento para los jóvenes, y algunas otras propuestas individuales que en conjunto buscan atacar sistemáticamente la problemática de dicho parque.

Los proyectos que se presentan son:

- Proyecto A. Estrategias emergentes de identidad para la vinculación de comunidades en la Alameda Norte'.

- Proyecto B. Estrategias emergentes para el caso del Ajolotario en el Parque Tezozómoc².

\subsection{Proyecto A. Estrategias emergentes de identidad para la vinculación de comunidades en la Alameda Norte}

Histórica y geográficamente, el término Alameda se ha utilizado para referirse a uno de los parques elegidos y nombrados de acuerdo con los puntos cardinales de la Ciudad de México (Alameda Sur, Alameda Central, Alameda Norte). Estos espacios se concibieron con el fin de crear un sentido de pertenencia a la Ciudad, en la que ninguno de sus puntos más alejado como el sur y el norte se sintiera excluido.

Con el paso del tiempo y la creciente mancha urbana, la necesidad de espacios verdes se multiplicó junto con la expansión de inmuebles, la oferta de parques aumentó y con ello, aquellos que histórica y geográficamentehabíanacompañado a la Ciudad de México desde sus inicios, se perdieron entre el concreto.

Hoy en día las opciones de espacios verdes en la Alcaldía Azcapotzalco son amplias. Muchos de los parques que actualmente perciben la mayoría de visitas por parte de los vecinos de la zona y su periferia es en su mayoría por la amplia oferta de experiencias y eventos, pero sobre todo de espacios dignos de apropiación ciudadana por su cuidado, constante limpieza y renovación de las áreas. Ante este escenario los alumnos proyectaron oportunidades de mejora en el contexto de la alameda, mismas que documentaron (Ver Figuras 2 y 3 ). 


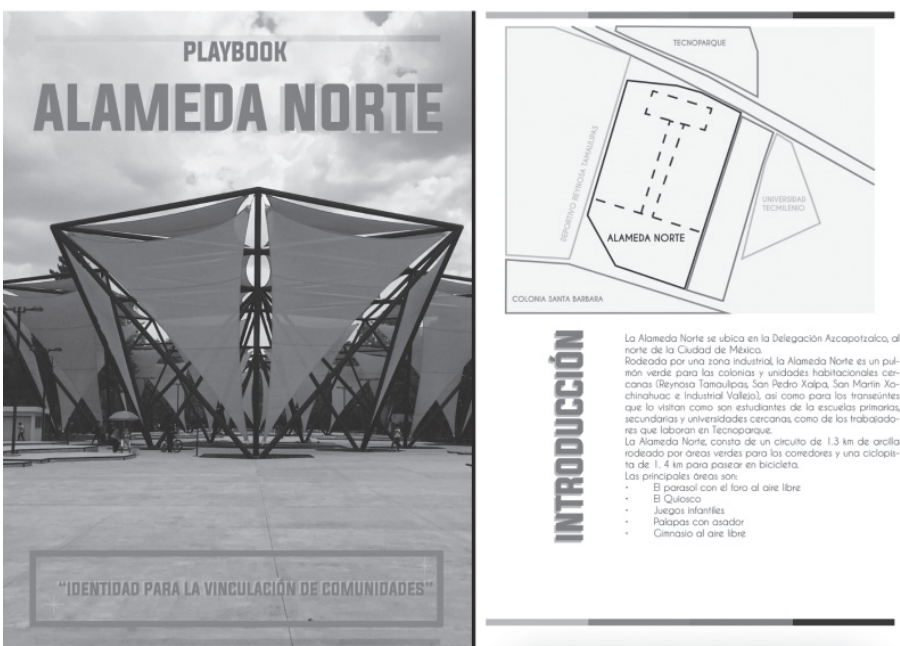

Figura 2. Alameda Norte (Material del curso elaborado por Daniela Osorio, Rocío Martínez, Mariel Sánchez, y Mitzi Alcántara).
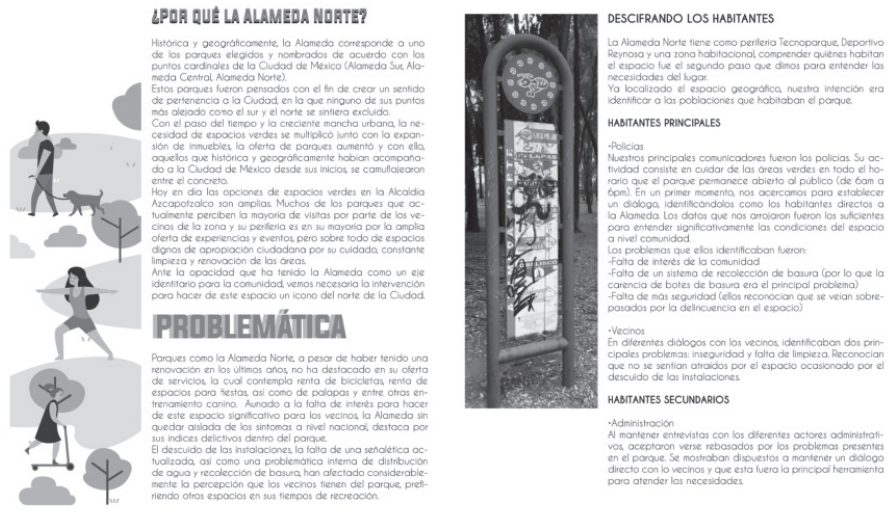

HABTANTES SECUNDRRIOS

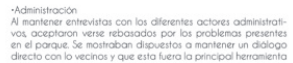

Figura 3. Productos de investigación (Material del curso elaborado por Daniela Osorio, Rocío Martínez, Mariel Sánchez, y Mitzi Alcántara).

Dicho parque contempla renta de bicicletas, renta de espacios para fiestas, así como de palapas y entrenamiento canino, entre otras. Aunado a la falta de interés para hacer de este espacio significativo para los vecinos, la Alameda sin quedar aislada de los síntomas a nivel nacional, destaca por sus índices delictivos dentro del parque. 
El descuido de las instalaciones, la falta de una señalética actualizada, así como una problemática interna de distribución de agua y recolección de basura, han afectado considerablemente la percepción que los vecinos tienen del parque, prefiriendo otros espacios en sus tiempos de recreación.

$\mathrm{Al}$ observar las condiciones del parque los alumnos buscaron identificar a los principales usuarios. Así mismo, notaron que había pocos niños aun cuando existen varias áreas asignadas para juegos infantiles. Todo esto quedó documentado en una primera investigación fotográfica. Los alumnos también documentaron la periferia del parque con lo que buscaban entender las poblaciones cercanas y mapear los actores que podrían estar involucrados.

Los problemas que dichos actores principales mencionaron fueron los siguientes:

- Falta de interés de la comunidad

- Falta de un sistema de recolección de basura (por lo que la carencia de botes de basura era el principal problema)

- Falta de más seguridad (ellos reconocían que se veíanrevasados por la delincuencia en el espacio)

En diferentes diálogos con los vecinos, identificaban dos principales problemas: inseguridad y falta de limpieza. Reconocían que no se sentíanatraídos por el espacio ocasionado por el descuido de las instalaciones.
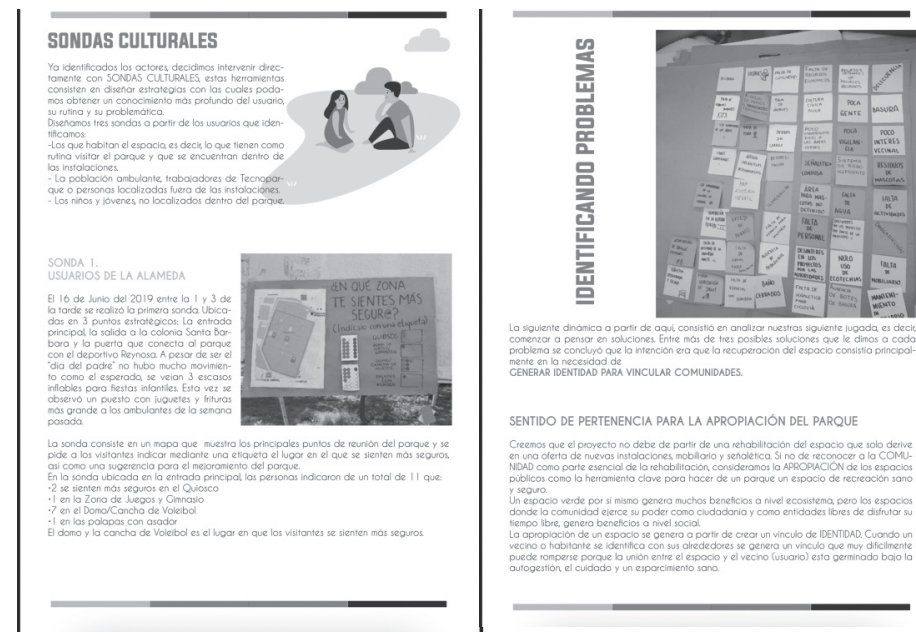

Figura 4. Productos de investigación (Material del curso elaborado por Daniela Osorio, Rocío Martínez, Mariel Sánchez, y Mitzi Alcántara). 
Una de las principales herramientas que los alumnos decidieron utilizar para entender a los asistentes del parque más a fondo fue la denominada sondas culturales. Ésta herramienta se recomienda a los estudiantes porque les permite recopilar información sin necesidad de estar presentes todo el tiempo, puede en algunos casos no ser muy invasiva al usuario y en general recopila información de mayor calidad que una simple entrevista. Se diseñaron tres sondas dirigidas a los principales usuarios identificados:

- Los que habitan el espacio, es decir, lo que tienen como rutina visitar el parque y que se encuentran dentro de las instalaciones.

- La población ambulante, trabajadores de la zona.

- Los niños y jóvenes, no localizados dentro del parque.

El principal problema detectado por los estudiantes fue la falta de sentido de pertenencia para la apropiación del parque. Lo cual ayudó a los estudiantes a desarrollar pensamiento crítico y formar una visión de lo que debería ser el parque. Bajo su perspectiva, éste parque no debía necesariamente buscar una rehabilitación del espacio que sólo derive en una oferta de nuevas instalaciones, mobiliario y señalética; más bien la propuesta debería tener como eje estratégico reconocer a la COMUNIDAD como parte esencial de la rehabilitación. Considerar la APROPIACIÓN de los espacios públicos como la herramienta clave para hacer de un parque un espacio de recreación sano y seguro.

En palabras de los propios alumnos "Un espacio verde por sí mismo genera muchos beneficios a nivel ecosistema, pero los espacios donde la comunidad ejerce su poder como ciudadanía y como entidades libres de disfrutar su tiempo libre, genera beneficios a nivel social." Durante las sesiones de revisión del proyecto se llegó a un acuerdo de que la apropiación de un espacio se genera a partir de crear un vinculo de IDENTIDAD. Cuando un vecino o habitante se identifica con sus alrededores se genera un vinculo que muy difícilmente puede romperse porque la unión entre el espacio y el vecino (usuario) esta germinado bajo la autogestión, el cuidado y un esparcimiento sano.

En las propuestas de los estudiantes para dicho espacio se buscaron formas de apoyar a la comunidad en su autogestión. A partir de estudiar los temas de innovación social y economías distribuidas en el aula de clases, los alumnos pudieron ver como la generación de comunidades autogestivas a nivel cultural son fundamentales para erradicar la violencia, es por ello que este proyecto busca rehabilitar espacios con el fin de que la motivación para visitar la Alameda Norte sea porque hay espacios de calidad donde se puedan reunir, divertir y APROPIAR DE LOS ESPACIOS. La propuesta estratégica tiene oportunidades de desarrollo de mobiliario, señalética, diseño de experiencias y de servicios.

En términos generales, la preocupación que expresan los alumnos a través de este ejercicio escolar es de rescatar el espacio público. Es fundamental volver a los parques y detonar actividades en la comunidad que puedan ejercer agencia cultural. El espacio público está vinculado a la calidad de vida de la ciudad.

La autogestión abona al tejido social nuevos métodos de hacer ciudadanía, por lo que es fundamental para este proyecto confiar en ella. Los eventos como los bazares y eventos artísticos, así como los recreativos y deportivos, pueden reactivar las economías locales, abonando a la creación de una identidad en el espacio y generación de nuevos vínculos sociales. 


\subsection{Proyecto B. Estrategias emergentes para el caso del Ajolotario en el Parque Tezozómoc}

Este proyecto representa un esfuerzo por rescatar al ajolote debido a que está en peligro de extinción. Como parte del ejercicio escolar, sus autores diseñaron una serie de estrategias con la finalidad de generar un impacto positivo en los visitantes del sitio en cuestión. La idea es generar un ambiente agradable en donde quepa la posibilidad de conocer más a fondo al ajolote mexicano. Se trata de generar conciencia medioambiental e involucrar a las personas a ser un agente de cambio de conciencia.

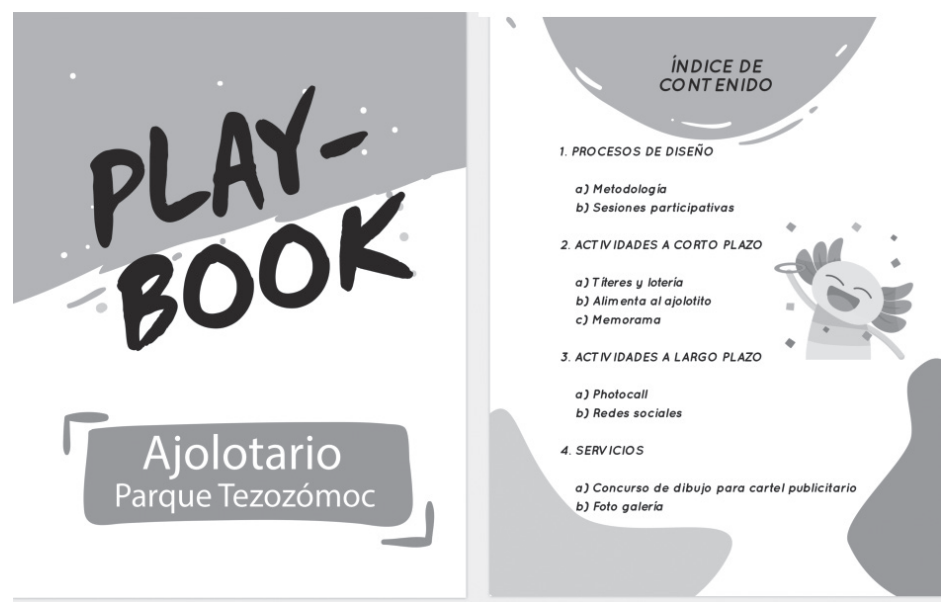

Figura 5. Ajolotario Parque Tezozómoc (Material del curso elaborado por Isaías De la Torre, Eduardo Maffey, Carlos Rodríguez, Alain Salas, y Brenda Venegas).

Los alumnos desarrollaron un ideario a manera de manifiesto el cual clarifica una visión que generan en equipo, la cual pretende ser transversal a todas sus acciones para fundar una estrategia que mantenga el espíritu de todas sus propuestas. Este manifiesto ayuda a mantenerlos objetivos claros y al equipo unido bajo una misma misión.

De igual manera, realizaron entrevistas a los agentes principales involucrados con el espacio denominado Ajolotario. Dentro de estos agentes se encuentran biólogos que realizan prácticas en el lugar, personal administrativo y el público en general. Ellos, orientaron respecto a los procesos requeridos para continuar con el proyecto en el parque además de comentar problemáticas que ya se tenían detectadas.

Uno de los hallazgos más importante sobre el Ajolotario del parque Tezozómoc fue su falta de identidad. El lugar no cuenta con presencia digital por lo que no hay una referencia que los usuarios puedan tener previamente al asistir a la zona. Los alumnos comienzan a mostrar un interés de promover las actividades y los objetivos del Ajolotario del parque 
Tezozómoc para mejorar la experiencia de los usuarios facilitando el cumplimiento de su misión de educar y divulgar información respecto al Ajolote Mexicano.

Otra de las herramientas utilizadas en este curso fue una sesión intensiva de lluvia de ideas la cual fue facilitada por el profesor en una serie de referencias culturales que se pudieran adaptar al proyecto.

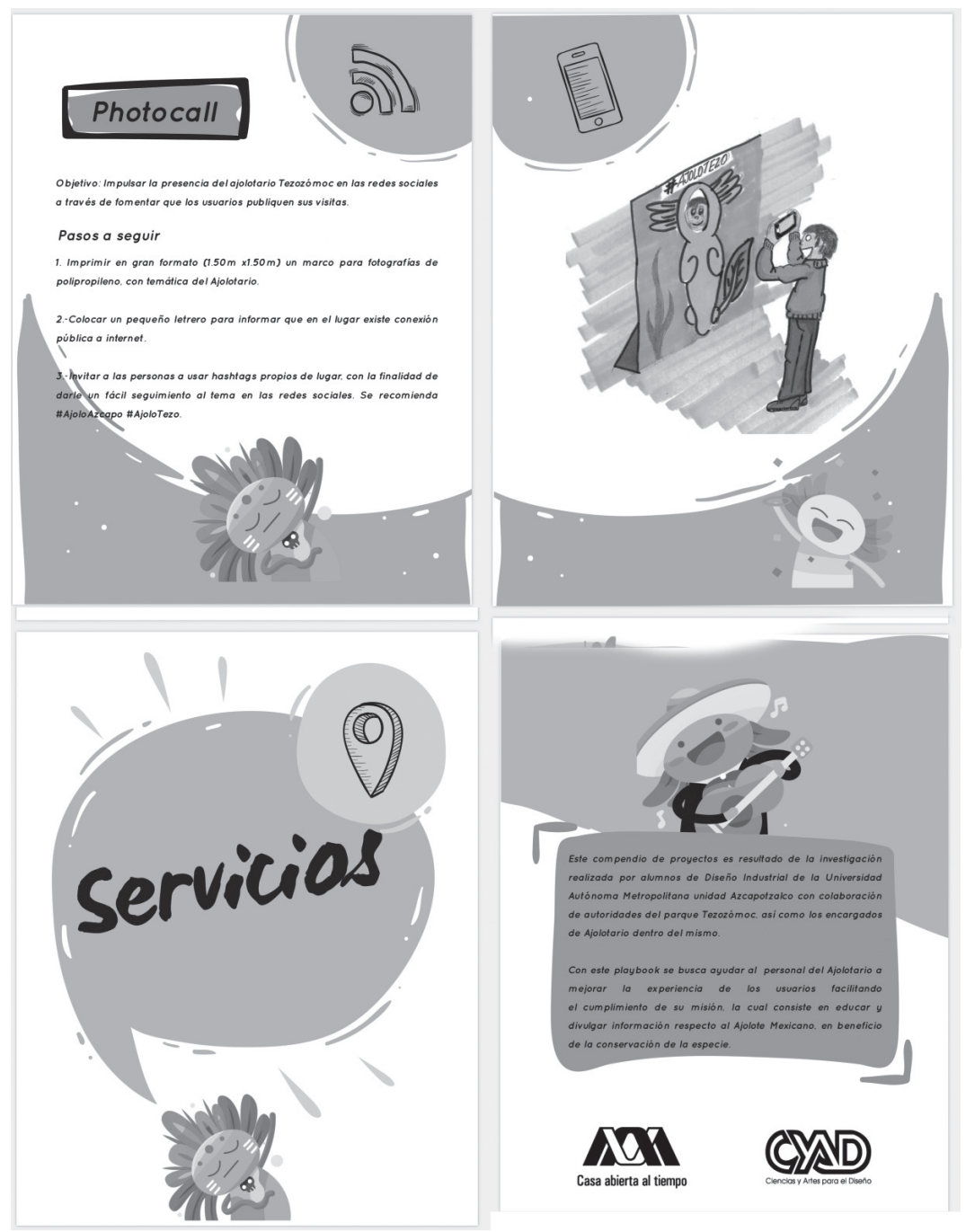

Figura 6. Soluciones propuestas para el proyecto integradas en un Playbook. 
Las soluciones elaboradas forman parte de un playbook, entendido como un documento que contiene una serie de estrategias que buscan ayudar al personal del Ajolotario a mejorar la experiencia de los usuarios facilitando el cumplimiento de su misión, la cual consiste en educar y divulgar información respecto al Ajolote Mexicano, en beneficio de la conservación de la especie.

\section{Conclusiones}

La descripción de los proyectos de diseño presentados en este artículo ha servido para reconocer que, si bien algunos de los elementos fundamentales del concepto de economías distribuidas están presentes en el acto proyectual del diseño, no necesariamente son un espejo de este modelo alternativo de desarrollo industrial sustentable. Lo mismo pasa con los conceptos de estrategia emergente y pensamiento exponencial. Se deja entrever en los proyectos de los estudiantes la horizontalidad para involucrar a todos los actores posibles en el proceso de diseño o la importancia de la calidad de vida, por citar un par de ejemplos. Sin embargo, no es tan evidente la presencia de otros aspectos derivados de los conceptos de pensamiento exponencial o estrategia emergente.

Se reconoce la necesidad de establecer una estructura que facilite a los diseñadores aplicar los principios de una economía distribuida, teniendo en consideración también los elementos para una estrategia emergente y un pensamiento exponencial que favorezcan el desarrollo sustentable.

Dicha estructura puede entenderse como un PlayBook que sirve de guía a los estudiantes de diseño o diseñadores profesionales para proyectar mejores sistemas de productoservicio sustentables.

Una línea de investigación a futuro consistiría en proponer una manera estructurada de aplicar los conceptos de economía distribuida, estrategia emergente y pensamiento exponencial a lo largo de diversos cursos que aborden problemas complejos que busquen promover el desarrollo de soluciones estratégicas.

\section{Bibliografía}

Atkinson, Paul (2011) "Orchestral Manouvers in Design" en Open Design Now. Bas van Abel, Lucas Evers, Roel Klassen, y Peter Troxler. BIS Publishers, Premsela, Waag Society. PaísesBajos.

Boal, Augusto (1998) Legislative Theater. Routledge. New York, USA.

Brown, A. M. (2017). Emergent Strategy: Shaping Change, Changing Worlds.

Diamandis, P., Kotler, S. (2015). BOLD: How to go big, create wealth, and impact the world. Simon \& Schuster Paperbacks. (2016 ed.)

Ezio Manzini “Design, When Everybody Designs” MIT Press, USA, 2015

Escobar, Arturo. (2018) Designs for the Pluriverse: Radical Independence, Autonomy, and the Making of Worlds. Duke University Press. 
Hoffmann, A. (2018). Impact. In Purpose \& Impact (p. 256). Retrieved from https://lccn. loc.gov/2017054536

Johansson, A., Kisch, P., \&Mirata, M. (2005). Distributed economies - A new engine for innovation. Journal of Cleaner Production, 13(10-11), 971-979. doi:10.1016/j.jclepro.2004.12.015

Kumar, Vijay (2013) 101 Design Methods. Ed. Wiley. USA.

Lipsod, Hod. "Fabricated: The New World of 3d Printing" (2013)

Lizarralde, I., \&Tyl, B. (2018). A framework for the integration of the conviviality concept in the design process. Journal of Cleaner Production, 197, 1766-1777. doi:10.1016/j. jclepro.2017.03.108

Margolin, Victor. The Politics of the Artificial: Essays on Design and Design Studies. (Chicago: University of Chicago Press, 2002)

Sörman-Nilsson, A. (2013). Digilogue : how to win the digital minds and analogue hearts of tomorrow's customers (2013 ed.).

Thackara, John. 2004. In the Bubble: Designing in a Complex World. Cambridge, MZ. MIT Press.

\begin{abstract}
Designing for people, their environment and their situation also means changing the approach towards human beings, their experiences and their context. It is necessary to go from a change in unconscious development, that is, only due to increased productivity, to conscious considering the cycles of the elements and their impact in the future. Design careers likewise have undergone a shift in focus from object to subject. The problems' complexity gives designers more possibilities of development, but the limits of the discipline are more blurred. This work explores the link between design education and distributed economy based on the concepts proposed by Ezio Manzini, Brown's emerging theory, and Diamandis' exponential thinking. Because of that, several design exercises and experiences are reviewed. The intention is to achieve a better understanding of how these concepts contribute to projecting more strategic design solutions ad hoc for new development models such as distributed economies.
\end{abstract}

Keywords: Design Careers - design object - design subject - distributed economy

Resumo: Projetar para as pessoas, seu ambiente e situação também significa mudar a abordagem das coisas para os seres humanos, suas experiências e seu contexto. É necessário uma mudança no desenvolvimento inconsciente, ou seja, devido ao aumento da produtividade, ao consciente considerando os ciclos dos elementos e seu impacto no futuro. Da mesma forma, as carreiras de design passaram por uma mudança de foco no objeto para no assunto. A complexidade dos problemas oferece ao projetista mais possibilidades de desenvolvimento, mas os limites da disciplina são mais difusos. Este trabalho explora a ligação entre a educação em design e a economia distribuída, com base 
nos conceitos propostos por Ezio Manzini, a teoria emergente de Brown e o pensamento exponencial de Diamandis. Para isso, várias experiências de exercícios de design são revisadas. A intenção é entender melhor como esses conceitos contribuem para projetar soluções de design mais estratégicas ad hoc para novos modelos de desenvolvimento, como economias distribuídas.

Palavras chave: Carreiras de design - objeto de design - assunto do design - economia distribuída

[Las traducciones de los abstracts fueron supervisadas por el autor de cada artículo] 
